\title{
Data Clarification Form
}

National Cancer Institute

\section{Source}

National Cancer Institute. Data Clarification Form. NCI Thesaurus. Code C115521.

Documentation used to query database discrepancies and record approved corrections to a clinical trial database. 